\title{
Relationship of vocal cord paralysis to the coil diameter of vagus nerve stimulator leads
}

\author{
Leslie C. Robinson, MD, PharmD, MBA, ${ }^{1}$ and Ken R. Winston, MD ${ }^{1-4}$ \\ ${ }^{1}$ Department of Neurosurgery, University of Colorado School of Medicine, Aurora; ${ }^{2}$ Denver Health and Hospitals, Denver; \\ ${ }^{3}$ University Hospital; and ${ }^{4}$ Children's Hospital Colorado, Aurora, Colorado
}

\begin{abstract}
OBJECT This investigation was done to examine, following implantation of vagus nerve stimulators, the relationship of vocal cord paralysis to the inner diameter of the coils used to attach the stimulator lead to the nerve.

METHODS All data in this investigation were collected, as mandated by the FDA, by the manufacturer of vagus nerve stimulators and were made available without restrictions for analysis by the authors. The data reflect all initial device implantations in the United States for the period from 1997 through 2012.

RESULTS Vocal cord paralysis was reported in 193 of 51,882 implantations. In patients aged 18 years and older, the incidence of paralysis was $0.26 \%$ when the stimulator leads had coil diameters of $3 \mathrm{~mm}$ and $0.51 \%$ when the leads had 2 -mm-diameter coils $(p<0.05)$. Across all age groups, the incidence of vocal cord paralysis increased with age at implantation for leads having 2-mm-diameter coils.
\end{abstract}

CONCLUSIONS In patients aged 18 years and older, vocal cord paralysis occurred at almost twice the rate with the implantation of vagus nerve stimulator leads having 2-mm-diameter coils than with leads having 3-mm-diameter coils. The incidence of vocal cord paralysis increases with patient age at implantation.

http://thejns.org/doi/abs/10.3171/2014.10.JNS14640

KEY WORDS complications; epilepsy; vocal cord paralysis; vagus nerve injury; vagus nerve stimulator lead

$\mathrm{V}$ OCAL cord paralysis is a serious and much-feared complication related to the implantation of vagus nerve stimulators. Strong evidence supports the effectiveness of vagus nerve stimulation (VNS) in reducing the frequency and severity of seizures in many patients with epilepsy and in the treatment of other disorders, such as depression and intractable hiccups. ${ }^{17-9}$ Implantation of vagus stimulators is less invasive than other operations done for epilepsy and has a lower incidence of associated complications, particularly the more serious ones. ${ }^{2} \mathrm{Com}-$ plications from the surgery required for implantation include failure to obtain benefit, infection, and permanent vocal cord paralysis. ${ }^{4,10,11,13}$ An increasing number of new implantations are done in the United States each year, and vocal cord paralysis is reported to occur in approximately $1 \%$ of these cases. ${ }^{3,5,10,14}$ Although vocal cord paralysis occurs uncommonly, this risk must be explained to patients when obtaining informed consent and, unfortunately, frightens some patients away from trying VNS therapy. Information that could reduce this risk and any associated fear would be important.

The coils that secure the stimulator lead to the vagus nerve are available in two inner diameters: 2 and $3 \mathrm{~mm}$. We performed this study to test the hypothesis that vocal cord paralysis following implantation of vagus nerve stimulators occurs more frequently with leads having the smaller 2-mm coils than those with 3-mm coils.

\section{Methods}

All data for this investigation were made available to us by Cyberonics Inc., the manufacturer of vagus nerve stimulators. The FDA requires the manufacturer to collect and annually report adverse events that have occurred within 6 months after the implantation of vagus nerve stimulators. The data requested and made available for this study 
reflect all initial implantations and all instances of vocal cord paralysis recorded in the manufacturer's registry of implants in the United States for the period from 1997 through 2012. Criteria for the diagnosis of vocal cord paralysis in patients in this database were not available for analysis. Nonetheless, all diagnoses of vocal cord paralysis were accepted as being accurate for this study.

No financial support, no monetary reward of any kind, and no promise or understanding of future reward was received from the manufacturer. Their representatives exercised no input or influence in the investigational design, analysis and interpretation of data, or conclusions drawn. Neither did they have any right of approval, alteration, or rejection of the prepared manuscript prior to its submission for publication.

The 1-tailed chi-square statistic with Yates correction was used to assess probabilities in $2 \times 2$ tables under consideration. A binomial distribution was assumed for the computation of $95 \%$ confidence intervals (http://statpages. org/confint.html). A p value $\leq 0.05$ was required for significance.

\section{Results}

From 1997 through 2012 in the United States, 51,882 initial implants of vagus nerve stimulators were registered with the manufacturer, and vocal cord paralysis was reported in 193 cases $(0.37 \%$; $95 \%$ CI $0.32-0.43)$ within 6 months following implantation. The age span for the patients was 11-87 years. No information regarding the resolution of vocal cord paralysis was available. Ninetysix percent of the patients who were younger than 18 years and $90 \%$ of those who were 18 years or older had received the leads with 2 -mm coils ( $\mathrm{p}<0.01)$. Vocal cord paralysis was reported in $184(0.38 \%)$ of the 47,746 implants having the 2-mm-diameter coils and in $9(0.22 \%)$ of the 4136 implants having the 3-mm-diameter coils. The age distribution in vocal cord paralysis comparing the two coil diameters is shown in Table 1 . Vocal cord paralysis was more often associated with the 2-mm coils in all age groups. It occurred in $0.49 \%$ of all patients who were 18 years or older but was nearly twice as common in those with the $2-\mathrm{mm}$ coils $(0.51 \%)$ than in those with the $3-\mathrm{mm}$ coils $(0.26 \%$; $p<0.05)$. None of the patients younger than 18 years or older than 64 years had vocal cord paralysis following implantation of a lead having 3-mm coils. Nine-

TABLE 1. Distribution of vocal cord paralysis in patients with implanted vagus nerve stimulators, as a function of coil diameter on stimulator leads

\begin{tabular}{lcc}
\hline & \multicolumn{2}{c}{ Coil Diameter } \\
\cline { 2 - 3 } \multicolumn{1}{c}{ Parameter } & $2 \mathrm{~mm}$ & $3 \mathrm{~mm}$ \\
\hline Total no. of cases & 47,746 & 4136 \\
\hline Patients w/ age $<18$ yrs & $\mathrm{p}>0.05$ & \\
\hline No. of implants & 15,533 & 687 \\
\hline No. of vocal cord paralysis cases & $19(0.12 \%)$ & $0(0.00 \%)$ \\
\hline Patients w/ age $\geq 18$ yrs & $p<0.05$ & \\
\hline No. of implants & 32,213 & 3449 \\
\hline No. of vocal cord paralysis cases & $165(0.51 \%)$ & $9(0.26 \%)$ \\
\hline
\end{tabular}

teen of the 15,533 who were 18 years or younger had this problem with the 2-mm coils (Fig. 1). For patients with the 3 -mm coils, the $0.20 \%$ incidence of vocal cord paralysis in the 18-34 years age group was not significantly different from the $0.31 \%$ incidence in the 35-64 years age group $(\mathrm{p}=0.74)$. The incidence of vocal cord paralysis following new implantations increased with advancing age for patients who had received the 2-mm coils but not for those who had received the 3-mm coils. No difference was identified in the incidence of vocal cord paralysis between males and females, nor was there a relationship between vocal cord paralysis and coil sizes within either patient sex. No trend in the occurrence of vocal cord paralysis was identified across the years of 1997 through 2012.

\section{Discussion}

Patients 18 years of age or older experienced a significantly $(\mathrm{p}<0.05)$ higher incidence of vocal cord paralysis following initial implantation of vagus nerve stimulator leads having coil diameters of $2 \mathrm{~mm}(0.51 \%)$ than did patients whose leads had coil diameters of $3 \mathrm{~mm}(0.26 \%)$. No statistically significant relationship between vocal cord paralysis and coil size was identified in patients younger than 18 years $(\mathrm{p}>0.38)$; however, all 19 cases of vocal cord paralysis in this younger age group had 2-mm leads (Table 1). Only 9 instances of vocal cord paralysis occurred among the 4136 patients who had received 3-mm coils in the United States, and none of these cases occurred in the 687 patients who were younger than 18 years. Given the rarity of vocal cord paralysis occurring after implantations of vagus nerve stimulators $(0.37 \%)$, it is not surprising that the association between vocal cord paralysis and the smaller coil has not come to attention earlier.

Vocal cord paralysis that follows the implantation of a vagus nerve stimulator must occur from injury to the vagus nerve or to the recurrent laryngeal nerve. It is reasonable to believe that the vagus nerve is the site of injury in most if not all cases because 1) the paralysis occurs significantly more frequently with one coil size, 2) the surgical approach and details of the technique for surgical implantation are the same for both coil sizes, and 3) the recurrent laryngeal nerve is never knowingly disturbed.

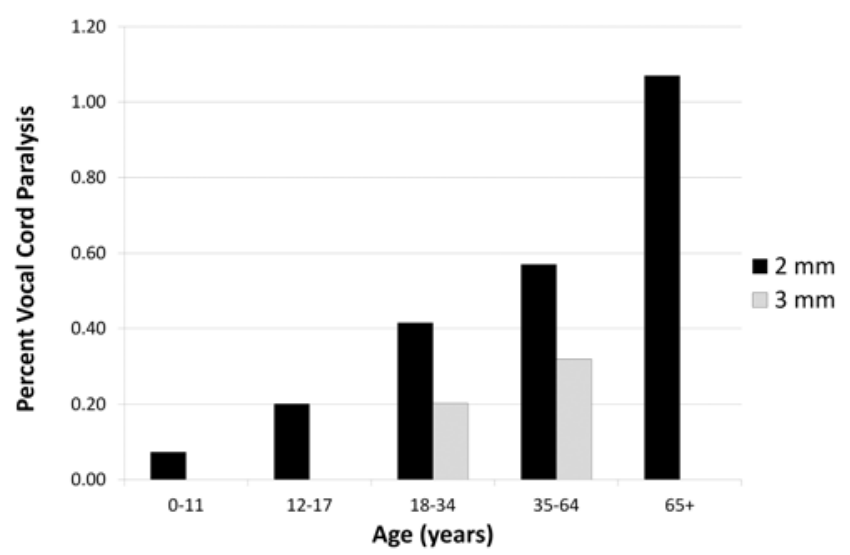

FIG. 1. Comparison of patient age groups having vocal cord paralysis following implantation of vagus nerve stimulators with leads having 2- or 3-mm coils. 
An anomalous nonrecurrent inferior laryngeal nerve can be present either with or without an aberrant subclavian artery, and perhaps such an anomaly could predispose the vagus nerve to injury during stimulator implantation..$^{2} \mathrm{We}$ have no data on the range of diameters, cross-sectional areas, or cross-sectional shapes of the vagus nerve at various patient ages, but it is reasonable to believe that there is variation in all of these categories in all age groups, with a tendency for the nerve to be smaller in children. After the nerve is surgically exposed, surgeons subjectively assess its size and choose for implantation a lead with either 2- or 3-mm coils. Although the basis for choosing the coil size may be inconsistent over time and from surgeon to surgeon, it is not surprising that leads with 2-mm coils are chosen significantly more frequently $(\mathrm{p}<0.01)$ in patients younger than 18 years $(96 \%)$ than in those aged 18 years and older (90\%).

The commonly assumed cause of vocal cord paralysis in these cases is surgical injury to the vagus nerve during stimulator implantation. Certainly the nerve can be injured while exposing and dissecting the nerve and then attaching the coils by compression with a hemostat or forceps, by heat and desiccation from electrocoagulation, from longitudinal stretching during dissection, or from complete transection. But these mechanisms alone cannot explain the identified difference in risk for the two coil sizes.

Another plausible explanation for vocal cord paralysis following implantation of a vagus nerve stimulator is constriction of the nerve as the result of a mismatch in size between a relatively large nerve and the surrounding helical coils. It would be difficult if not impossible for a surgeon to distinguish between a slightly snug fit that could be safely tolerated and a tighter fit that would cause immediate or delayed injury to the vagus nerve. Regardless of its crosssectional shape, when a nerve is tightly encircled by helical coils, it is forced into an annular shape, and each coil, acting as an elastic spring, applies relentless constriction. Following a seemingly uneventful and safe placement of coils, tight encirclement of the vagus nerve may produce immediate nerve injury and dysfunction or can result in ischemia, perhaps mild at first, with slowly progressing edema, tighter constriction, and worsening ischemia, until the axons that innervate the vocal cord are temporarily or irreversibly injured. The time course could be quite variable. Constriction of the vagus nerve with progressive ischemia could explain the delayed occurrence of vocal cord paralysis following implantation. ${ }^{6}$

The physical properties of the coils themselves must also be considered. The structural thickness of both 2- and $3-\mathrm{mm}$ coils are the same, but their radii of curvature, 1 and $1.5 \mathrm{~mm}$, respectively, are quite different. The smaller coils can be expected to have greater rigidity. Thus, a $2-\mathrm{mm}$ coil exerts greater constricting or closing force than does a 3-mm coil. Stated differently, the resistance to centrifugal expansion of a constricted nerve, whether slight or severe, is greater by $2-\mathrm{mm}$ coils than by $3-\mathrm{mm}$ coils. Snug encirclement of the vagus nerve within $2-\mathrm{mm}$ coils could be expected to be less well tolerated than that within the 3-mm coils.

The psychomotor task of dealing with coils that are smaller and more rigid requires greater manipulation of both the coils and the nerve. Although the surgical implantation of both 2- and 3-mm coils is typically understood and described as being insignificantly different, it is not uncommon for surgeons to find implantation of the smaller coils to be recognizably more difficult, more time consuming, and frustrating. The risk of inflicting injury to the vagus nerve during surgery is therefore increased as a result of the greater difficulty in attaching the smaller and structurally stiffer 2-mm coils. All surgeons are not equally experienced, and it is reasonable to believe that not all surgeons are equally adept at implanting vagus nerve stimulators. It is also possible that only a few surgeons across the country are responsible for a disproportionate number of nerve injuries in this series, but both of these possibilities seem very unlikely in our opinion, and there is no evidence to support either of them.

A steady increase in the incidence of vocal cord paralysis, from $0.07 \%$ in the youngest pediatric group to $1.07 \%$ in patients 65 years and older, was associated with the $2-\mathrm{mm}$ coils but not the 3-mm coils (Fig. 1). No case of vocal cord paralysis was reported with the 3-mm coils in any person younger than 18 years or over 64 years. A satisfactory explanation for the steadily increasing age-related vulnerability to injury of the vagus nerve in response to surgical implantation of only the 2-mm coils is not readily apparent. The vagus nerve is not known to enlarge throughout life nor in response to long-term exposure to anticonvulsant medications. A progressively increasing risk of injury to the nerve during surgical implantation that would increase across all age groups, both children and adults, seems unlikely. Interestingly, $92 \%$ of all implanted leads have $2-\mathrm{mm}$ coils - the rate is $90 \%$ for patients 65 years and older. Regardless of the reason, the implantation of leads with 2-mm coils is less well tolerated with advancing age, whether attributable to the structure of the coils themselves, the act of attaching them to the vagus nerve, or simply a diminishing tolerance by the nerve.

The global $0.37 \%$ incidence (95\% CI $0.32-0.43$ ) of vocal cord paralysis in this data set is lower than the incidences in several much smaller published series, ranging from $0 \%$ to $1.4 \%$; however, the $95 \%$ confidence intervals for these smaller series range from a low of $0 \%$ to a high of $7.3 \% .^{3,10,14}$ We recognize that an unknown number, probably small, of stimulators have been implanted without the knowledge of the manufacturer and that some instances of vocal cord paralysis were probably not reported. Criteria used in diagnosing vocal cord paralysis in the patients in this series were not available. Nonetheless, it is reasonable to believe that the patients identified as having vocal cord paralysis had symptoms and signs that were convincing, nontransient, and based on opinions from physicians who were chiefly, if not totally, epileptologists, neurosurgeons, or perhaps otolaryngological surgeons. It is also possible that vocal cord paralysis was missed in a very few patients, for example, those with tracheostomies, those with severely impaired vocal ability including some with preoperative vocal cord paralysis, and perhaps some young children. However, unilateral vocal cord paralysis can have significant effects on airflow, particularly in very young children. Surgeons, epileptologists, representatives of the manufacturer, and perhaps even patients report vo- 
cal cord paralysis to the manufacturer for a variety of reasons, not the least of which is the medicolegal concern. Regardless of the thoroughness with which vocal cord paralysis is reported, it is unlikely that unreported cases in this very large data set would be distributed significantly differently with respect to coil size. Prior to this report, there would have been no reason to suspect that vocal cord paralysis was related to coil size.

We are aware of no data describing a relationship between coil size and effectiveness in achieving seizure control, and it is not intuitively apparent that there should be a relationship. Choosing a coil that is $0.5 \mathrm{~mm}$ different in its inner radius would result in only a very small difference in the distance between the most remote axons within an encircled nerve and some portion of the encircling electrode. Even a very small nerve would never remain perfectly centered within the coils, except when there is a snug fit of the two electrode-containing coils. Scar tissue would fill any gaps between electrodes and nerve and is not an electrical insulator. There would always be electrode contact on much of the nerve, even small nerves.

Implantation of a vagus nerve stimulator is remarkably safe compared with all other operations done for epilepsy, and the incidence of vocal cord paralysis is small in all reports, including this one. ${ }^{3,4}$ A complication of vocal cord paralysis does not exclude the possibility of improved seizure control, and patients who benefit from their stimulation may consider vocal cord paralysis, while undoubtedly disturbing, to be worth the price. But the cost of permanent vocal cord paralysis for patients who have no improvement in seizure control must be especially disheartening. It is incumbent on surgeons to minimize this risk in all patients.

\section{Conclusions}

Implantation of vagus nerve stimulators with leads having 2-mm inner-diameter coils in patients 18 years of age and older was associated with vocal cord paralysis at almost twice the rate as occurred with 3-mm inner-diameter coils: $0.51 \%$ versus $0.26 \%$. The risk of vocal cord paralysis is directly related to patient age at implantation. We argue that the $2-\mathrm{mm}$ coils are more rigid than the $3-\mathrm{mm}$ coils given their difference in radius of curvature, and this difference in rigidity, combined with the greater difficulty in attaching smaller stiffer coils to the vagus nerve, increases the risk of injury. Vocal cord paralysis is a rare but very serious complication, and every reasonable precaution should be made to avoid it in every patient.

\section{Acknowledgment}

We gratefully acknowledge Cyberonics Inc., particularly Clinton Wright, for providing the data, which made this report possible.

\section{References}

1. Alexopoulos AV, Kotagal P, Loddenkemper T, Hammel J,
Bingaman WE: Long-term results with vagus nerve stimulation in children with pharmacoresistant epilepsy. Seizure 15:491-503, 2006

2. Ben-Menachem E: Vagus nerve stimulation, side effects, and long-term safety. J Clin Neurophysiol 18:415-418, 2001

3. Handforth A, DeGiorgio CM, Schachter SC, Uthman BM, Naritoku DK, Tecoma ES, et al: Vagus nerve stimulation therapy for partial-onset seizures: a randomized active-control trial. Neurology 51:48-55, 1998

4. Kahlow H, Olivecrona M: Complications of vagal nerve stimulation for drug-resistant epilepsy: a single center longitudinal study of 143 patients. Seizure 22:827-833, 2013

5. Kalkanis JG, Krishna P, Espinosa JA, Naritoku DK: Selfinflicted vocal cord paralysis in patients with vagus nerve stimulators. Report of two cases. J Neurosurg 96:949-951, 2002

6. Landy HJ, Ramsay RE, Slater J, Casiano RR, Morgan R: Vagus nerve stimulation for complex partial seizures: surgical technique, safety, and efficacy. J Neurosurg 78:26-31, 1993

7. Longatti P, Basaldella L, Moro M, Ciccarino P, Franzini A: Refractory central supratentorial hiccup partially relieved with vagus nerve stimulation. J Neurol Neurosurg Psychiatry 81:821-822, 2010 (Erratum in J Neurol Neurosurg Psychiatry 82:236, 2011)

8. Milby AH, Halpern $\mathrm{CH}$, Baltuch $\mathrm{GH}$ : Vagus nerve stimulation for epilepsy and depression. Neurotherapeutics 5:7585, 2008

9. Payne BR, Tiel RL, Payne MS, Fisch B: Vagus nerve stimulation for chronic intractable hiccups. Case report. J Neurosurg 102:935-937, 2005

10. Smyth MD, Tubbs RS, Bebin EM, Grabb PA, Blount JP: Complications of chronic vagus nerve stimulation for epilepsy in children. J Neurosurg 99:500-503, 2003

11. Spitz MC, Winston KR, Maa EH, Ojemann SG: Insulation discontinuity in a vagus nerve stimulator lead: a treatable cause of intolerable stimulation-related symptoms. J Neurosurg 112:829-831, 2010

12. Tateda M, Hasegawa J, Sagai S, Nakanome A, Katagiri K, Ishida E, et al: Nonrecurrent inferior laryngeal nerve without vascular anomaly as a genuine entity. Tohoku J Exp Med 216:133-137, 2008

13. Tran Y, Shah AK, Mittal S: Lead breakage and vocal cord paralysis following blunt neck trauma in a patient with vagal nerve stimulator. J Neurol Sci 304:132-135, 2011

14. Vagus Nerve Stimulation Study Group: A randomized controlled trial of chronic vagus nerve stimulation for treatment of medically intractable seizures. Neurology 45:224-230, 1995

\section{Author Contributions}

Conception and design: Winston. Analysis and interpretation of data: both authors. Drafting the article: both authors. Critically revising the article: both authors. Reviewed submitted version of manuscript: both authors. Approved the final version of the manuscript on behalf of both authors: Winston. Study supervision: Winston.

\section{Correspondence}

Ken R. Winston, 777 Bannock St., Denver, CO 80204. email: krwinston@gmail.com. 